\title{
Voxelwise Relationships Between Distribution Volume Ratio and Cerebral Blood Flow: Implications for Analysis of $\beta$-Amyloid Images
}

\author{
Jitka Sojkova ${ }^{1}$, Joshua Goh ${ }^{1,2}$, Murat Bilgel ${ }^{1,3}$, Bennett Landman ${ }^{4}$, Xue Yang ${ }^{4}$, Yun Zhou ${ }^{5}$, Yang An ${ }^{1}$, \\ Lori L. Beason-Held ${ }^{1}$, Michael A. Kraut ${ }^{5}$, Dean F. Wong ${ }^{5-8}$, and Susan M. Resnick ${ }^{1}$ \\ ${ }^{1}$ Laboratory of Behavioral Neuroscience, National Institute on Aging, NIH, Bethesda, Maryland; ${ }^{2}$ National Taiwan University, Taipei, \\ Taiwan; ${ }^{3}$ Department of Biomedical Engineering, Johns Hopkins University, Baltimore, Maryland; ${ }^{4}$ Department of Electrical \\ Engineering, Vanderbilt University, Nashville, Tennessee; ${ }^{5}$ Russell H. Morgan Department of Radiology and Radiological Sciences, \\ Johns Hopkins University, Baltimore, Maryland; ${ }^{6}$ Department of Psychiatry and Behavioral Sciences, Johns Hopkins University, \\ Baltimore, Maryland; ${ }^{7}$ Solomon Snyder Department of Neuroscience, Johns Hopkins University, Baltimore, Maryland; and \\ ${ }^{8}$ Environmental Health Sciences, Johns Hopkins University, Baltimore, Maryland
}

Quantification of $\beta$-amyloid $(A \beta)$ in vivo is often accomplished using the distribution volume ratio (DVR), based on a simplified reference tissue model. We investigated the local relationships between DVR and cerebral blood flow (CBF), as well as relative $C B F\left(R_{1}\right)$, in nondemented older adults. Methods: Fifty-five nondemented participants (mean age, $78.5 \mathrm{y}$ ) in the Baltimore Longitudinal Study of Aging underwent ${ }^{15} \mathrm{O}-\mathrm{H}_{2} \mathrm{O}$ PET $\mathrm{CBF}$ and dynamic ${ }^{11} \mathrm{C}-\mathrm{PiB}$ PET. ${ }^{15} \mathrm{O}-\mathrm{H}_{2} \mathrm{O}$ PET images were normalized and smoothed using SPM. A simplified reference tissue model with linear regression and spatial constraints was used to generate parametric DVR images. The DVR images were regressed on CBF images on a voxel-by-voxel basis using robust biologic parametric mapping, adjusting for age and sex (false discovery rate, $P=0.05$; spatial extent, 50 voxels). DVR images were also regressed on $R_{1}$ images, a measure of the transport rate constant from vascular space to tissue. All analyses were performed on the entire sample, and on high and low tertiles of mean cortical DVR. Results: Voxel-based analyses showed that increased DVR is associated with increased CBF in the frontal, parietal, temporal, and occipital cortices. However, this association appears to spare regions that typically show early $A \beta$ deposition. $A$ more robust relationship between DVR and CBF was observed in the lower tertile of DVR, that is, negligible cortical $A \beta$ load, compared with the upper tertile of cortical DVR and $A \beta$ load. The spatial distributions of the DVR-CBF and DVR-R $_{1}$ correlations showed similar patterns. No reliable negative voxelwise relationships between DVR and CBF or $R_{1}$ were observed. Conclusion: Robust associations between DVR and CBF at negligible $A \beta$ levels, together with similar spatial distributions of DVR-CBF and DVR- $R_{1}$ correlations, suggest that regional distribution of DVR reflects blood flow and tracer influx rather than pattern of $A \beta$ deposition in those with minimal $A \beta$ load. DVR-CBF associations in individuals with a higher DVR are more likely to reflect true associations between patterns of $A \beta$ deposition and CBF or neural activity. These findings have important implications for analysis and interpretation of voxelwise correlations with external variables in individuals with varying amounts of $A \beta$ load.

Received Nov. 11, 2014; revision accepted Apr. 15, 2015.

For correspondence or reprints contact: Susan M. Resnick, Laboratory of Behavioral Neuroscience, Biomedical Research Center, National Institute on Aging, IRP, 251 Bayview Blvd., Room 4B335, Baltimore, MD 21224.

E-mail: resnicks@mail.nih.gov

Published online May 14, 2015.

COPYRIGHT (C 2015 by the Society of Nuclear Medicine and Molecular Imaging, Inc.
Key Words: amyloid; cerebral blood flow; PiB; aging; DVR

J Nucl Med 2015; 56:1042-1047

DOI: $10.2967 /$ jnumed.114.151480

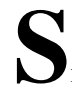
several studies have investigated $\mathrm{A} \beta$ deposition in nondemented older adults using ${ }^{11} \mathrm{C}$-Pittsburgh compound B (PiB) (2-6). Accurate quantification of $A \beta$ is especially important in this population, where associations with other biomarkers and individual differences in cognition and genetic, metabolic, and other medical covariates may enhance understanding of the pathophysiology and temporal sequence of changes in Alzheimer disease (AD), including the timing of $A \beta$ deposition in relation to changes in brain structure and function (7).

An inverse association between $\mathrm{A} \beta$ deposition and regional cerebral glucose metabolism has been demonstrated in $\operatorname{AD}(1,8,9)$. However, recent findings suggest that deficits in cerebral glucose metabolism, measured by ${ }^{18} \mathrm{~F}$-FDG PET, may not always match the regional distribution of $\mathrm{A} \beta$ deposition. For example, in atypical clinical variants of $\mathrm{AD}$, such as the logopenic variant of primary progressive aphasia and posterior cortical atrophy, the pattern of glucose hypometabolism is consistent with the clinical presentation of these $A D$ variants despite similarly widespread $A \beta$ deposition by the time of clinical diagnosis (10). The association between $A \beta$ deposition and glucose metabolism is even less clear in nondemented older adults, with studies yielding conflicting results (1113). Because of considerations of participant burden and cost, it is not always feasible to perform both ${ }^{11} \mathrm{C}$-PiB PET and ${ }^{18} \mathrm{~F}$-FDG PET on the same individual. Measures of regional cerebral blood flow (CBF) obtained with ${ }^{15} \mathrm{O}-\mathrm{H}_{2} \mathrm{O}$ PET provide an alternative for investigation of regional brain function that is more readily integrated into an ${ }^{11} \mathrm{C}$-PiB PET study because of the short ${ }^{15} \mathrm{O}$ half-life and imaging duration.

In addition, dynamic acquisition of ${ }^{11} \mathrm{C}-\mathrm{PiB}$ PET studies allows estimation of parameters that may reflect both $\mathrm{A} \beta$ and $\mathrm{CBF}$. For example, the simplified reference tissue model (SRTM) with linear regression and spatial constraint (14) provides estimates of the 
distribution volume ratio (DVR) for detection of $A \beta$ and an estimate of $R_{1}$ (relative $C B F$ ). $R_{1}$ is the target-to-reference tissue ratio of the tracer transport rate constant from vascular space to tissue $\left(K_{1} / K_{1 \text { ref }}\right)(14)$ and correlates well with regional cerebral glucose metabolism, measured by ${ }^{18} \mathrm{~F}-\mathrm{FDG}$ PET (15). As such, $\mathrm{R}_{1}$ can be used to enhance our understanding of the relationship between regional brain function and $A \beta$ deposition in nondemented older adults on whom ${ }^{18} \mathrm{~F}$-FDG PET is not performed.

In this study, we first investigated the voxelwise association of ${ }^{11} \mathrm{C}-\mathrm{PiB}$ PET measures of $\mathrm{A} \beta$ deposition with concurrently acquired CBF measurements obtained from ${ }^{15} \mathrm{O}-\mathrm{H}_{2} \mathrm{O}$ PET in nondemented participants in the Baltimore Longitudinal Study of Aging. Next, we used the same approach to examine the relationship between the DVR measure of $A \beta$ burden and the $R_{1}$ estimate of CBF based on the same ${ }^{11} \mathrm{C}$-PiB PET dynamic studies. We hypothesized that in nondemented older adults, DVR would be positively correlated with $\mathrm{CBF}$ and that this relationship would vary with the spatial distribution of $A \beta$ deposition. Second, we expected that spatial patterns of associations between DVR and $\mathrm{R}_{1}$ would parallel those between DVR and ${ }^{15} \mathrm{O}-\mathrm{H}_{2} \mathrm{O}$ measures of $\mathrm{CBF}$.

\section{MATERIALS AND METHODS}

\section{Participants}

The participants were 55 nondemented adults (mean age $\pm \mathrm{SD}$, $78.5 \pm 6.3 \mathrm{y}$ ) from the neuroimaging substudy of the Baltimore Longitudinal Study of Aging (16) who underwent ${ }^{11} \mathrm{C}-\mathrm{PiB}$ PET and resting ${ }^{15} \mathrm{O}-\mathrm{H}_{2} \mathrm{O}$ PET on the same day. Seven of the participants had a Clinical Dementia Rating Scale (17) score of 0.5, 3 of whom met consensus conference-based criteria for mild cognitive impairment at the time of the study. Consensus diagnosis followed established procedures $(18,19)$ and was based on serial neuropsychologic evaluations and the Clinical Dementia Rating Scale score, which was typically informant-based. The neuropsychologic measures used for consensus diagnosis included tests of mental status, word knowledge, verbal ability, memory, language, verbal fluency, attention, executive function, and visuospatial ability. Mild cognitive impairment was diagnosed in participants with progressive decline in a single cognitive domain or in more than a single domain with absence of documented functional impairment. Dementia was diagnosed according to the criteria of the Diagnostic and Statistical Manual of Mental Disorders III-R (20), and $\mathrm{AD}$ was diagnosed according to the criteria of the National Institute of Neurologic and Communicative Disorders and Stroke-Alzheimer's
Disease and Related Disorders Association (21). Exclusionary criteria included stroke or head trauma. Sample characteristics are shown in Table 1.

This study was approved by the Institutional Review Boards of the National Institute on Aging Intramural Research Program and the Johns Hopkins Medicine institutions. Written informed consent was obtained from each participant at each visit.

\section{${ }^{11} \mathrm{C}-\mathrm{PiB}$ PET Image Acquisition and Processing}

Dynamic ${ }^{11} \mathrm{C}$-PiB PET studies were performed on an Advance scanner (GE Healthcare) in 3-dimensional mode, and 33 time frames (70-min acquisition) were obtained. Image acquisition started immediately after intravenous bolus injection of $539.5 \pm 30.0 \mathrm{MBq}(14.58 \pm$ $0.81 \mathrm{mCi}$ ) of ${ }^{11} \mathrm{C}-\mathrm{PiB}$ with a specific activity of $205.7 \pm 110.3 \mathrm{GBq}$ $(5.56 \pm 2.98 \mathrm{Ci}) / \mu \mathrm{mol}$. Participants were fitted with a thermoplastic mask to minimize motion during scanning. Transmission scans in 2-dimensional mode using a ${ }^{68} \mathrm{Ge}$ source were used for attenuation correction. Dynamic images were reconstructed using filtered backprojection with a ramp filter (image size, $128 \times 128$; pixel size, $2 \times$ $2 \mathrm{~mm}$; slice thickness, $4.25 \mathrm{~mm}$ ), yielding a spatial resolution of about $4.5 \mathrm{~mm}$ in full width at half maximum at the center of the field of view.

MR images, coregistered to the corresponding ${ }^{11} \mathrm{C}-\mathrm{PiB}$ PET images, were used to define ROIs. Spoiled gradient recalled or magnetizationprepared rapid-acquisition gradient echo MR images (124 slices; image matrix, $256 \times 256$; pixel size, $0.94 \times 0.94 \mathrm{~mm}$; slice thickness, $1.5 \mathrm{~mm}$ ) were coregistered to the mean of the first $20 \mathrm{~min}$ of dynamic PET images for each participant using the mutual information method in the Statistical Parametric Mapping software (SPM2; Wellcome Department of Cognitive Neurology). With the exception of a single claustrophobic participant whose structural MR images were obtained $10 \mathrm{y}$ before the initial ${ }^{11} \mathrm{C}-\mathrm{PiB}$ PET assessment, participants underwent structural MR imaging within $3 \mathrm{y}$ of each ${ }^{11} \mathrm{C}-\mathrm{PiB}$ PET study. The cerebellar gray-matter ROI, which was used as the reference region, was manually drawn on the MR images and then applied to the ${ }^{11} \mathrm{C}$-PiB PET images. In addition to the ROI on the cerebellum, 15 other ROIs were manually drawn on the coregistered MR images to sample the PET radioactivity $(14,22)$.

Parametric DVR images were generated by simultaneous fitting of an SRTM with linear regression and spatial constraint to dynamic ${ }^{11} \mathrm{C}$ PiB PET images $(14,23)$. DVRs for the 15 ROIs were then extracted from the parametric images. Mean cortical DVR (cDVR) was calculated by averaging DVRs from the orbitofrontal, prefrontal (including middle and inferior frontal gyri), superior frontal, parietal, lateral

\section{TABLE 1}

Demographic, Genetic, and Cognitive Data

\begin{tabular}{|c|c|c|c|c|c|}
\hline Parameter & Whole sample & Lower tertile & Middle tertile & Upper tertile & $P$ \\
\hline Total participants $(n)$ & 55 & 18 & 19 & 18 & \\
\hline Age (y) & $78.5(6.3)$ & $78.3(6.8)$ & $77.4(6.7)$ & $79.8(5.4)$ & $0.34^{*}$ \\
\hline Male participants $(n)$ & $31(56.4 \%)$ & $11(61.1 \%)$ & 7 (36.8\%) & $13(72.2 \%)$ & $0.10^{\dagger}$ \\
\hline White participants $(n)$ & $48(87.3 \%)$ & $17(94.4 \%)$ & $14(73.7 \%)$ & 17 (94.4\%) & $0.19^{\dagger}$ \\
\hline Education (y) & $16.9 \pm 2.3$ & $16.4 \pm 2.3$ & $17.6 \pm 1.8$ & $16.6 \pm 2.6$ & $0.23^{*}$ \\
\hline $\mathrm{CDR}=0.5(n)$ & $7(12.7 \%)$ & $0(0 \%)$ & $2(10.5 \%)$ & $5(27.8 \%)$ & 0.04 \\
\hline ApoE4 carrier $(n)$ & $16(29.1 \%)$ & $1(5.6 \%)$ & $5(26.3 \%)$ & $10(55.6 \%)$ & 0.004 \\
\hline \multicolumn{6}{|l|}{$\begin{array}{l}{ }^{*} \text { Kruskal-Wallis test. } \\
{ }^{\dagger} \text { Fisher exact test. } \\
\text { CDR = Clinical Dem }\end{array}$} \\
\hline
\end{tabular}


temporal, occipital, and anterior and posterior cingulate regions. Parametric images were then spatially normalized using an $\mathrm{R}_{1}\left(K_{1} / K_{1 \text { ref }}\right)$ template (14) and smoothed with a gaussian filter of 8,8 , and $8 \mathrm{~mm}$ in the $x, y$, and $z$ planes, respectively. We defined minimal cDVR as DVRs below 1.062 , based on the test-retest variability for DVR of $\pm 6.2 \%$ using SRTM analysis (24) and the fact that a DVR of 1 was considered to denote absence of specific binding.

\section{${ }^{15} \mathrm{O}-\mathrm{H}_{2} \mathrm{O}$ PET Acquisition and Image Processing}

Resting-state ${ }^{15} \mathrm{O}-\mathrm{H}_{2} \mathrm{O}$ scans were acquired on an Advance PET scanner in 3-dimensional mode. Images were obtained for $60 \mathrm{~s}$ once the total radioactivity counts in the brain reached threshold levels, as described previously (25). A transmission scan in 2-dimensional mode using a ${ }^{68} \mathrm{Ge}$ rotating source was applied for attenuation correction. ${ }^{15} \mathrm{O}-\mathrm{H}_{2} \mathrm{O}$ scanning was performed after the transmission scan and before the ${ }^{11} \mathrm{C}-\mathrm{PiB}$ PET scan, with $10 \mathrm{~min}$ allowed between scans.

Using Statistical Parametric Mapping (SPM5), ${ }^{15} \mathrm{O}-\mathrm{H}_{2} \mathrm{O}$ PET scans were spatially normalized into standard stereotaxic space and smoothed using a gaussian kernel to a full width at half maximum of 10,10 , and $10 \mathrm{~mm}$ in the $x, y$, and $z$ planes, respectively. To control for variability in global flow, rCBFs at each voxel were ratio-adjusted to the mean global flow and scaled to $50 \mathrm{~mL} / 100 \mathrm{~g} / \mathrm{min}$ for each image.

\section{Voxelwise Relationship of DVR with CBF and $\mathbf{R}_{\mathbf{1}}$}

We used Robust Biologic Parametric Mapping (robust BPM), version $2.1(26,27)$, to determine voxelwise DVR-CBF and DVR- $\mathrm{R}_{1}$ relationships. BPM allows voxelwise regression of data from 2 imaging modalities using least-squares regression (28). Robust BPM extends this approach by using robust regression (M-estimation) (29) to account for outliers in the imaging data (26).

We first regressed DVR images on globally adjusted CBF images, using age and sex as covariates, in the entire sample of 55 participants. We then repeated the regression analyses for the upper $(n=18)$ and lower $(n=18)$ tertiles defined by cDVR. Subsequently, we regressed DVR images on $R_{1}$ images, using age and sex as covariates, both in the whole group and in the upper- and lower-tertile subgroups defined by cDVR. Significant effects for all analyses were generated, with false-discovery-rate correction at $P=0.05$ and a spatial extent of more than 50 voxels.

\section{RESULTS}

\section{Cortical ${ }^{11} \mathrm{C}-\mathrm{PiB}$ Retention}

The distribution of CDVR by tertile is shown in Figure 1. The mean cDVRs were $1.17 \pm 0.26$ for the entire sample, $0.93 \pm 0.02$ (range, 0.89-0.97) in the lower tertile, and $1.49 \pm 0.16$ (range, 1.25-1.76) in the upper tertile. The DVRs for the 9 cortical ROIs comprising the cDVR are presented in Table 2 for the whole sample, as well as for the upper- and lower-tertile subgroups. As expected from the subgroup definition, the upper and lower tertiles showed significant differences for cDVR and regional DVRs $(P<0.005)$.

\section{Association Between DVR and ${ }^{15} \mathrm{O}-\mathrm{H}_{2} \mathrm{O}$ CBF}

In the entire sample, only positive correlations were observed between DVR and CBF using a conservative threshold of 0.05 for the false discovery rate and a spatial extent of 50 voxels. With a higher DVR and greater amyloid burden, a higher CBF was seen in the frontal (left superior and left middle frontal gyrus), parietal (right and left paracentral lobule and postcentral gyri, right superior parietal lobule), temporal (right and left superior temporal gyrus and uncus, right inferior temporal gyrus), and occipital (right

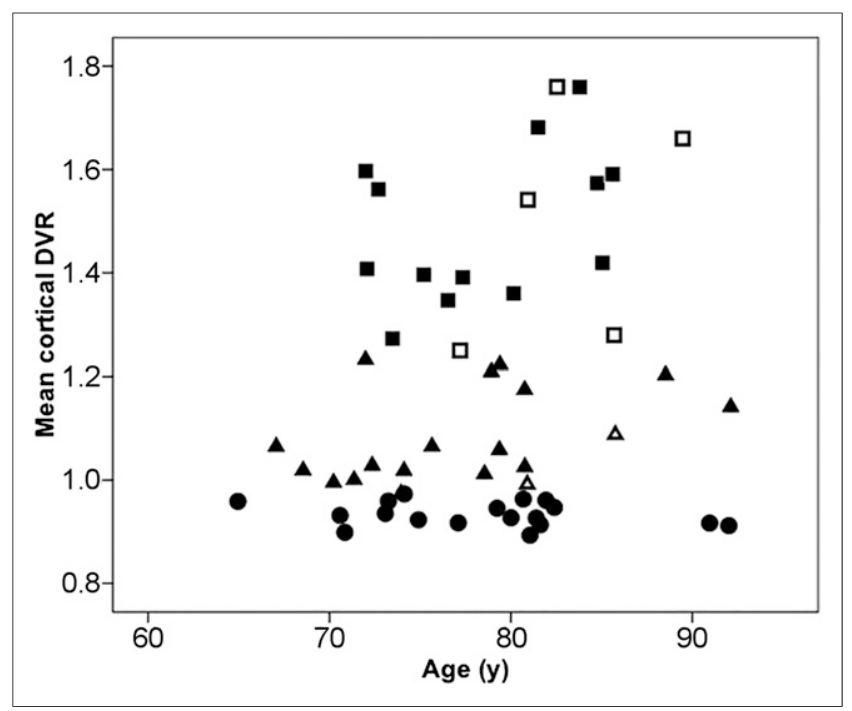

FIGURE 1. Distribution of cDVRs by tertile. Squares denote upper tertile; triangles, middle tertile; circles, lower tertile. Solid symbols denote individuals with Clinical Dementia Rating Scale score of 0; open symbols, individuals with score of 0.5 .

cuneus) cortices, as well as in the right and left thalamus, right midbrain, and right and left cerebellum (Supplemental Table 1; supplemental materials are available at http://jnm.snmjournals.org). Of the cortical structures, the largest spatial extent (4,772 voxels) and T-value (11.16) were localized to the paracentral lobule. Figure 2A illustrates DVR-CBF relationships in the paracentral lobule, with extension primarily anterior to the medial frontal gyrus. Importantly, DVR was not related to CBF in the orbitofrontal gyrus, in most of the cingulate gyrus, or in the precuneus, although these regions have moderate to high amounts of $A \beta$ (Table 2). Nevertheless, there are also regions, such as the superior frontal gyrus, that have an elevated DVR $(1.2 \pm 0.34)$ and do show an association between DVR and CBF. Interestingly, the medial temporal gyrus, which has a very low DVR (1.01 \pm 0.09$)$, showed robust voxelwise relationships between DVR and $\mathrm{CBF}$.

We next investigated the relationship between DVR and CBF by cDVR tertile. We found that a higher DVR was associated with a higher $\mathrm{CBF}$ in the upper-tertile subgroup. These findings were localized to the frontal (left superior, middle, and orbitofrontal gyri, right medial and inferior frontal gyri, right and left subcallosal gyrus), parietal (right paracentral lobule), temporal (right and left superior temporal gyrus, left middle temporal gyrus, right fusiform gyrus, left insula), and occipital (left middle occipital gyrus, left cuneus) lobes, as well as to the right cingulate gyrus, left caudate, and right cerebellum (Supplemental Table 1). The greatest spatial extent (1,237 voxels), with a T-value of 16.61 , was in the right paracentral lobule. Figure 2A shows associations between DVR and $\mathrm{CBF}$ in the medial frontal gyrus and the paracentral lobule for the upper-tertile subgroup.

DVR-CBF analysis in the lower-tertile subgroup showed greater spatial extent than in the upper-tertile subgroup (Fig. 2A). DVR$\mathrm{CBF}$ correlations were localized to the frontal (right and left superior frontal gyrus, right inferior frontal gyrus, left precentral gyrus), parietal (right inferior parietal lobule), and temporal (right superior, middle, and inferior temporal gyri, right fusiform gyrus, right uncus) cortices, as well as to the corpus callosum and cerebellum 
TABLE 2

Global and Regional DVRs

\begin{tabular}{lcccc}
\hline \multicolumn{1}{c}{ DVR } & Whole sample & Lower tertile & Middle tertile & Upper tertile \\
\hline Number of participants & 55 & 18 & 19 & 18 \\
\hline Mean cDVR & $1.17 \pm 0.26^{*}$ & $0.93 \pm 0.02$ & $1.08 \pm 0.09^{*}$ & $1.49 \pm 0.16^{\star}$ \\
\hline Orbitofrontal cortex & $1.09 \pm 0.27$ & $0.86 \pm 0.04$ & $0.1 \pm 0.14$ & $1.40 \pm 0.19$ \\
\hline Prefrontal cortex & $1.11 \pm 0.29$ & $0.86 \pm 0.04$ & $1.02 \pm 0.13$ & $1.46 \pm 0.19$ \\
\hline Superior frontal gyrus & $1.20 \pm 0.34$ & $0.90 \pm 0.04$ & $1.08 \pm 0.14$ & $1.61 \pm 0.22$ \\
\hline Parietal lobe & $1.14 \pm 0.24$ & $0.92 \pm 0.05$ & $1.07 \pm 0.08$ & $1.42 \pm 0.19$ \\
\hline Medial temporal cortex & $1.01 \pm 0.09$ & $0.95 \pm 0.07$ & $1.01 \pm 0.06$ & $1.06 \pm 0.11$ \\
\hline Lateral temporal cortex & $1.13 \pm 0.24$ & $0.93 \pm 0.03$ & $1.05 \pm 0.12$ & $1.41 \pm 0.19$ \\
\hline Occipital cortex & $1.08 \pm 0.13$ & $0.99 \pm 0.06$ & $1.06 \pm 0.07$ & $1.19 \pm 0.15$ \\
\hline Anterior cingulate gyrus & $1.27 \pm 0.34$ & $0.97 \pm 0.07$ & $1.17 \pm 0.18$ & $1.67 \pm 0.23$ \\
\hline Posterior cingulate gyrus & $1.32 \pm 0.35$ & $1.02 \pm 0.06$ & $1.19 \pm 0.1$ & $1.76 \pm 0.23$ \\
\hline
\end{tabular}

${ }^{*}$ Greater than DVR positivity cutoff of 1.062 for cDVR, indicating more than minimal A.

All DVRs are significantly higher in upper tertile than lower tertile $(P<0.005)$.

(Supplemental Table 1). Of these regions, the corpus callosum had the greatest spatial extent (19,901 voxels) and T-value (20.24).

Several DVR-CBF associations were found across analyses for the upper- and lower-tertile subgroups. These include the left superior and inferior frontal gyri, right superior and middle temporal gyri, and right cingulate gyrus.

\section{Association Between DVR and $\mathbf{R}_{\mathbf{1}}$}

Similar to the findings for associations between DVR and ${ }^{15} \mathrm{O}-\mathrm{H}_{2} \mathrm{O} \mathrm{CBF}$ in the entire sample, only positive correlations between DVR and $\mathrm{R}_{1}$ reached the statistical threshold. DVR- $\mathrm{R}_{1}$ correlations were in regions similar to the DVR-CBF correlations: the frontal, parietal, temporal, and occipital cortices, as well as the cerebellum (Fig. 2B; Supplemental Table 2). The DVR-R 1 correlation of greatest extent was in the medial frontal gyrus, paracentral lobule, and cerebellum.

In addition, we investigated DVR and $\mathrm{R}_{1}$ by cDVR tertile. In both the upper- and the lower-tertile subgroups, only positive associations between $R_{1}$ and DVR were observed (Fig. $2 B$ ). In the upper tertile, DVR- $\mathrm{R}_{1}$ associations were localized to the medial frontal gyrus, paracentral lobule, thalamus, and cerebellum, and the spatial distribution of DVR- $\mathrm{R}_{1}$ associations was similar to but of greater spatial extent than that of DVR-CBF associations. Compared with the upper-tertile subgroup, the lower-tertile subgroup had a greater spatial extent of DVR- $\mathrm{R}_{1}$ associations, and these associations involved most of the frontal, parietal, temporal, and occipital cortices, as well as the thalamus and cerebellum. In the lower-tertile subgroup, spatial extent was greater for $\mathrm{DVR}-\mathrm{R}_{1}$ associations than for DVR-CBF associations.

\section{DISCUSSION}

Our voxelwise analysis of ${ }^{15} \mathrm{O}-\mathrm{H}_{2} \mathrm{O}$ and ${ }^{11} \mathrm{C}-\mathrm{PiB}$ PET in nondemented older adults revealed that positive relationships between local DVR and CBF are less pronounced in many regions that typically have high $\mathrm{A} \beta$ deposition. Overall, relationships between DVR and CBF are more robust in individuals with a low $A \beta$ load than in those with a high $A \beta$ load. Furthermore, associations between DVR and $R_{1}$ show patterns similar to those between
DVR and CBF. Specifically, individuals with a low DVR and low levels of $A \beta$ demonstrate more robust $D V R-R_{1}$ relationships than individuals with a high $\mathrm{A} \beta$ load. Together, these findings suggest that at low DVR levels, regional patterns in DVR images

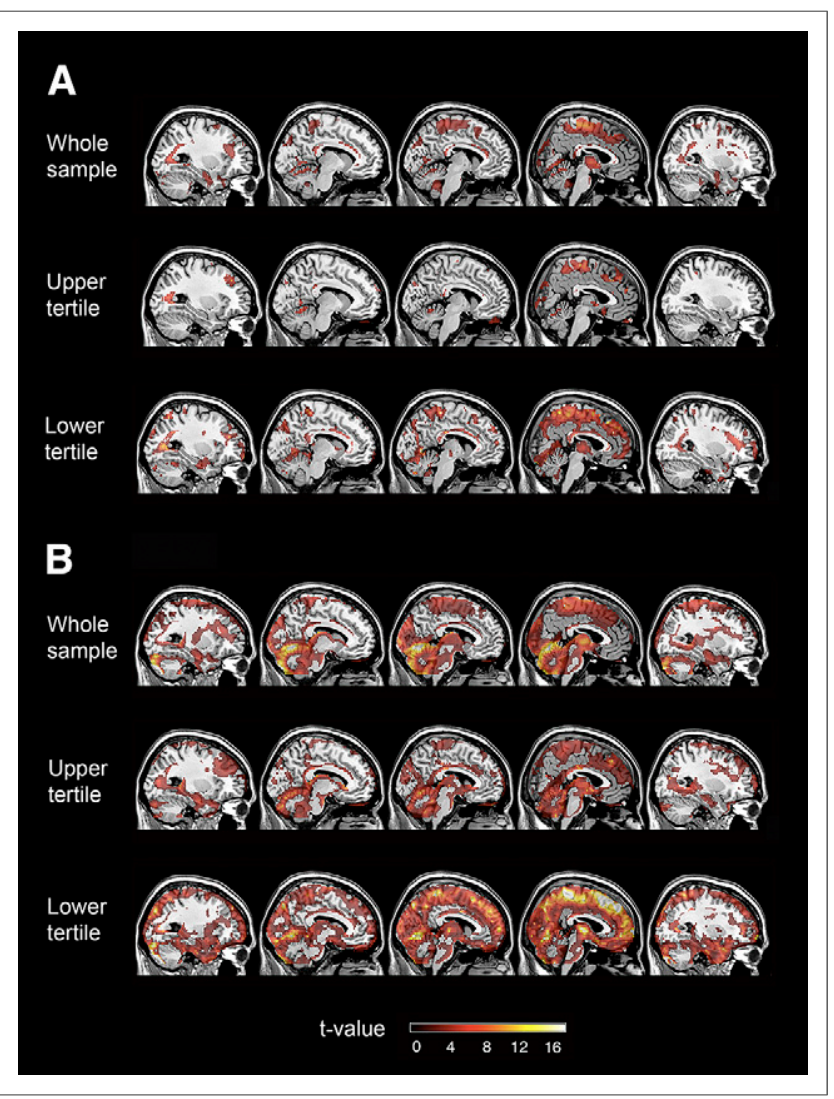

FIGURE 2. (A) Positive associations between CBF and DVR. (B) Positive associations between $R_{1}$ and DVR. 
reflect blood flow and tracer influx to tissue rather than reflecting $\mathrm{A} \beta$ deposition.

We found significant positive but no inverse associations between DVR and CBF in individuals without a clinical diagnosis of dementia. The spatial distribution of this DVR-CBF relationship with respect to the distribution of $A \beta$ load provides insight for understanding the DVR-CBF associations. A higher DVR is related to a higher CBF in the frontal, parietal, temporal, and occipital cortices, with the greatest spatial extent being in the paracentral lobule and extending anteriorly to the medial frontal gyrus. However, regions with early $A \beta$ deposition, such as the precuneus, do not show extensive and robust associations between DVR and CBF. Thus, there may be factors other than increased $A \beta$ load that underlie the DVR-CBF relationships in regions showing extensive associations in the sample as a whole.

To further investigate this possibility, we examined the effect of $A \beta$ load, measured by mean cDVR, on the relationship between DVR and CBF. We found that both the magnitude and the spatial extent of DVR-CBF relationships were greater in the lower-tertile subgroup, with negligible or low $A \beta$ load. As in the analysis of the whole sample, regions that typically have high amounts of $A \beta$ did not show extensive relationships between DVR and CBF. These findings suggest that DVR images at low cDVR levels likely represent patterns of $\mathrm{CBF}$ and tracer delivery, in addition to nonspecific binding, rather than $A \beta$ deposition. We also found that the region in the lower-tertile subgroup with the highest spatial extent for DVR-CBF association is the corpus callosum, consistent with delayed tracer clearance for ${ }^{11} \mathrm{C}-\mathrm{PiB}$ in white matter $(30)$.

To directly understand the contribution of tracer influx to DVR, we also investigated the relationship between DVR and $R_{1}$. We found a robust relationship involving most of the cortex between DVR and $R_{1}$ at low cDVR levels-a contrast to the lesser spatial extent of DVR- $R_{1}$ associations at high cDVR levels. This suggests that DVR images at low cDVR levels, in addition to reflecting blood flow, also reflect tracer influx. Nevertheless, as can be seen from Figure $2 B, R_{1}$ is related to DVR even in the upper tertile. Therefore, it is essential to determine the level of $A \beta$ load at which DVR images represent regional patterns of $A \beta$ deposition rather than blood flow or tracer influx. It is likely that differences in regional patterns of $\mathrm{PiB}$ retention at different levels of $\mathrm{A} \beta$ can be informative in this regard, as suggested by our recent report that spatial patterns of $\mathrm{PiB}$ retention and progression may be better markers of cognitive decline than is cDVR alone (31). A clearer understanding of the extent to which tracer influx contributes to regional patterns of $\mathrm{PiB}$ retention and other amyloid radiotracers will be important for both statistical analysis and clinical interpretation of amyloid imaging scans.

Both DVR- $\mathrm{R}_{1}$ and DVR-CBF associations were positive in direction, and regression of $\mathrm{R}_{1}$ on DVR localized to the medial frontal gyrus and the paracentral lobule, regions also showing DVR-CBF relationships. However, spatial extent was greater for DVR- $\mathrm{R}_{1}$ associations than DVR-CBF associations. In part, the greater correspondence between DVR and $R_{1}$ may reflect correlated noise, because $R_{1}$, unlike independently assessed $C B F$, is used to calculate the tissue concentration of the tracer (14). Our study extends the findings of Blomquist et al., who, in a study of a rhesus monkey, found that $K_{1}$ for ${ }^{11} \mathrm{C}-\mathrm{PiB}$ reflects $\mathrm{CBF}$ (32). Here, we have demonstrated similar, region-specific, patterns of DVR-CBF and DVR- $\mathrm{R}_{1}$ associations in humans.
Our study has important implications for interpretation of $A \beta$ imaging data. Voxelwise investigations of the relationship between local values of ${ }^{11} \mathrm{C}-\mathrm{PiB}$ and external variables may be misleading given that DVR images at lower cDVR levels may not represent $A \beta$ deposition but rather blood flow and tracer influx. Such analyses using genetic, cognitive, or other scalar data as covariates of interest assume that variation in regional distribution of tracer retention at both low and high levels reflects regional variation in $A \beta$ deposition. As we have shown, the distribution of DVRs at different mean DVR levels can represent different processes. With DVRs at higher cDVR levels reflecting regional patterns of $A \beta$ deposition while those at lower cDVR levels represent regional patterns of blood flow and tracer influx, correlation or regression analyses including images across $A \beta$ levels may lead to results that do not reflect a single biologic process. Although these findings are pertinent for investigations across diagnostic categories with a wide range of DVRs, they are perhaps more influential in nondemented older adults, a greater proportion of whom may have negligible levels of $A \beta$ load that might affect associations of DVR with external variables. On the other hand, this difference in the process underlying the spatial distribution of $\mathrm{PiB}$ retention at high and low overall levels of $A \beta$ deposition may improve classification between cognitively stable and cognitive declining individuals (31). These findings further underscore the importance of investigating the DVR cutoff at which images reflect factors other than $A \beta$ deposition.

In this study, we used robust BPM for DVR-CBF and DVR- $\mathrm{R}_{1}$ voxelwise correlations. An advance of robust BPM relative to standard BPM is its use of robust regression to reduce the effect of outliers that may be due to, for example, misregistration (33). In addition, we used SRTM to estimate DVR rather than using the widely applied Logan graphical analysis, because SRTM allows simultaneous estimation of DVR and $\mathrm{R}_{1}$. DVRs estimated by the Logan plot with reference tissue input are comparable to those estimated by SRTM at low noise levels of ROI kinetics but are underestimated in the presence of increased noise $(23,24)$. We also recognize that SUVR is commonly used for semiquantitative measurement of $\mathrm{PiB}$ binding, because of simplified data acquisition and quantification, and note that highly linear correlations between DVR and SUVR were reported in previous studies with low noise levels of ROI kinetics $(34,35)$. However, SUVR measurements are dependent on the time window of acquisition $(24,34)$, and bias relative to DVR estimation is correlated with magnitude (35). Therefore, correlations of SUVR with CBF or $R_{1}$ will vary with the time window of acquisition and the magnitude of amyloid deposition.

Our study had several limitations. It was limited to individuals without clinical diagnoses of dementia. Further investigation of DVR-CBF and DVR- $\mathrm{R}_{1}$ relationships in individuals with wider ranges of global DVRs such as seen in mild cognitive impairment or $\mathrm{AD}$ would allow for more direct evaluation of the cutoff of DVRs at which the regional patterns in the images are no longer heavily weighted by blood flow or tracer influx. In addition, although we excluded individuals with stroke or head trauma, other contributing factors that may relate to vascular disease were not considered. Nevertheless, our study also had several strengths. We studied individuals from the neuroimaging substudy of the BLSA, which represents a well-characterized sample of nondemented older adults followed for $20 \mathrm{y}$ (16). We also used robust BPM, which accounts for outliers, to provide more accurate estimates of intermodality correlations. 


\section{CONCLUSION}

We have demonstrated that at low levels of global ${ }^{11} \mathrm{C}-\mathrm{PiB}$ retention, the regional pattern of DVR likely reflects blood flow and influx of the tracer to tissue, in addition to nonspecific binding associated with tracer clearance. As such, studies investigating voxelwise regional relationships between DVR and scalar values, as well as imaging data, may be limited in interpretation unless the correlational analysis is restricted to individuals with more than minimal tracer retention and $A \beta$ load. On the other hand, differences in regional distribution of tracer retention at low and high levels of $A \beta$ may aid in the discrimination of cognitively normal individuals from impaired individuals.

\section{DISCLOSURE}

The costs of publication of this article were defrayed in part by the payment of page charges. Therefore, and solely to indicate this fact, this article is hereby marked "advertisement" in accordance with 18 USC section 1734 . This research was supported by the Intramural Research Program of the NIH and by National Institute on Aging research and development contracts HHSN260200400012C and K24 DA000412. No other potential conflict of interest relevant to this article was reported.

\section{ACKNOWLEDGMENTS}

We are grateful to the BLSA participants for their dedication to these studies. We also thank Wendy Elkins and Beth Nardi for managing the study, as well as the staff of the PET facility at Johns Hopkins University and the neuroimaging staff of the National Institute on Aging for their assistance. We thank Dr. Ramon Casanova for his assistance with BPM.

\section{REFERENCES}

1. Klunk WE, Engler H, Nordberg A, et al. Imaging brain amyloid in Alzheimer's disease with Pittsburgh Compound-B. Ann Neurol. 2004;55:306-319.

2. Aizenstein HJ, Nebes RD, Saxton JA, et al. Frequent amyloid deposition without significant cognitive impairment among the elderly. Arch Neurol. 2008;65:15091517 .

3. Mintun MA, Larossa GN, Sheline YI, et al. $\left[{ }^{11} \mathrm{C}\right] \mathrm{PIB}$ in a nondemented population: potential antecedent marker of Alzheimer disease. Neurology. 2006;67:446-452.

4. Resnick SM, Sojkova J, Zhou Y, et al. Longitudinal cognitive decline is associated with fibrillar amyloid-beta measured by [ $\left.{ }^{11} \mathrm{C}\right] \mathrm{PiB}$. Neurology. 2010;74:807-815.

5. Rowe CC, Ellis KA, Rimajova M, et al. Amyloid imaging results from the Australian Imaging, Biomarkers and Lifestyle (AIBL) study of aging. Neurobiol Aging. 2010;31:1275-1283.

6. Villemagne VL, Burnham S, Bourgeat P, et al. Amyloid beta deposition, neurodegeneration, and cognitive decline in sporadic Alzheimer's disease: a prospective cohort study. Lancet Neurol. 2013;12:357-367.

7. Jack CR Jr, Knopman DS, Jagust WJ, et al. Tracking pathophysiological processes in Alzheimer's disease: an updated hypothetical model of dynamic biomarkers. Lancet Neurol. 2013;12:207-216.

8. Edison P, Archer HA, Hinz R, et al. Amyloid, hypometabolism, and cognition in Alzheimer disease: an $\left[{ }^{11} \mathrm{C}\right] \mathrm{PIB}$ and $\left[{ }^{18} \mathrm{~F}\right] \mathrm{FDG}$ PET study. Neurology. 2007;68:501508

9. Engler H, Forsberg A, Almkvist O, et al. Two-year follow-up of amyloid deposition in patients with Alzheimer's disease. Brain. 2006;129:2856-2866.

10. Lehmann M, Ghosh PM, Madison C, et al. Diverging patterns of amyloid deposition and hypometabolism in clinical variants of probable Alzheimer's disease. Brain. 2013;136:844-858.
11. Cohen AD, Price JC, Weissfeld LA, et al. Basal cerebral metabolism may modulate the cognitive effects of Abeta in mild cognitive impairment: an example of brain reserve. J Neurosci. 2009;29:14770-14778.

12. Jagust WJ, Landau SM, Shaw LM, et al. Relationships between biomarkers in aging and dementia. Neurology. 2009;73:1193-1199.

13. Mosconi L, Rinne JO, Tsui WH, et al. Amyloid and metabolic positron emission tomography imaging of cognitively normal adults with Alzheimer's parents. Neurobiol Aging. 2013;34:22-34.

14. Zhou Y, Resnick SM, Ye W, et al. Using a reference tissue model with spatial constraint to quantify $\left[{ }^{11} \mathrm{C}\right]$ Pittsburgh compound B PET for early diagnosis of Alzheimer's disease. Neuroimage. 2007;36:298-312.

15. Meyer PT, Hellwig S, Amtage F, et al. Dual-biomarker imaging of regional cerebral amyloid load and neuronal activity in dementia with PET and ${ }^{11}$ C-labeled Pittsburgh compound B. J Nucl Med. 2011;52:393-400.

16. Resnick SM, Goldszal AF, Davatzikos C, et al. One-year age changes in MRI brain volumes in older adults. Cereb Cortex. 2000;10:464-472.

17. Morris JC. The Clinical Dementia Rating (CDR): current version and scoring rules. Neurology. 1993;43:2412-2414.

18. Driscoll I, Resnick SM, Troncoso JC, An Y, O'Brien R, Zonderman AB. Impact of Alzheimer's pathology on cognitive trajectories in nondemented elderly. Ann Neurol. 2006;60:688-695.

19. Kawas C, Gray S, Brookmeyer R, Fozard J, Zonderman A. Age-specific incidence rates of Alzheimer's disease: the Baltimore Longitudinal Study of Aging. Neurology. 2000;54:2072-2077.

20. American Psychiatric Association. Diagnostic and Statistical Manual of Mental Disorders: DSM-III-R. 3rd ed., rev. Washington, DC: American Psychiatric Association, 1987.

21. McKhann G, Drachman D, Folstein M, Katzman R, Price D, Stadlan EM. Clinical diagnosis of Alzheimer's disease: report of the NINCDS-ADRDA Work Group under the auspices of Department of Health and Human Services Task Force on Alzheimer's Disease. Neurology. 1984;34:939-944.

22. Price JC, Klunk WE, Lopresti BJ, et al. Kinetic modeling of amyloid binding in humans using PET imaging and Pittsburgh compound-B. J Cereb Blood Flow Metab. 2005;25:1528-1547.

23. Zhou Y, Endres CJ, Brasic JR, Huang SC, Wong DF. Linear regression with spatial constraint to generate parametric images of ligand-receptor dynamic PET studies with a simplified reference tissue model. Neuroimage. 2003;18:975-989.

24. Lopresti BJ, Klunk WE, Mathis CA, et al. Simplified quantification of Pittsburgh compound B amyloid imaging PET studies: a comparative analysis. J Nucl Med. 2005;46:1959-1972.

25. Beason-Held LL, Kraut MA, Resnick SM. I. Longitudinal changes in aging brain function. Neurobiol Aging. 2008;29:483-496.

26. Yang X, Beason-Held L, Resnick SM, Landman BA. Biological parametric mapping with robust and non-parametric statistics. Neuroimage. 2011;57:423430.

27. Yang X, Beason-Held L, Resnick SM, Landman BA. Robust biological parametric mapping: an improved technique for multimodal brain image analysis. Proc SPIE Int Soc Opt Eng. 2011;7962:79623X.

28. Casanova R, Srikanth R, Baer A, et al. Biological parametric mapping: a statistical toolbox for multimodality brain image analysis. Neuroimage. 2007;34:137143.

29. Fox J. Robust Regression: An R and S-PLUS Companion to Applied Regression. Thousand Oaks, CA: Sage Publications, Inc.; 2002.

30. Fodero-Tavoletti MT, Rowe CC, McLean CA, et al. Characterization of PiB binding to white matter in Alzheimer disease and other dementias. $J$ Nucl Med. 2009;50:198-204.

31. Yotter RA, Doshi J, Clark V, et al. Memory decline shows stronger associations with estimated spatial patterns of amyloid deposition progression than total amyloid burden. Neurobiol Aging. 2013;34:2835-2842.

32. Blomquist G, Engler H, Nordberg A, et al. Unidirectional influx and net accumulation of PIB. Open Neuroimag J. 2008;2:114-125.

33. Yang X, Lauzon CB, Crainiceanu C, Caffo B, Resnick SM, Landman BA. Biological parametric mapping accounting for random regressors with regression calibration and model II regression. Neuroimage. 2012;62:1761-1768.

34. McNamee RL, Yee SH, Price JC, et al. Consideration of optimal time window for Pittsburgh compound B PET summed uptake measurements. J Nucl Med. 2009;50:348-355

35. Zhou Y, Sojkova J, Resnick SM, Wong DF. Relative equilibrium plot improves graphical analysis and allows bias correction of standardized uptake value ratio in quantitative ${ }^{11} \mathrm{C}-\mathrm{PiB}$ PET studies. J Nucl Med. 2012;53:622-628. 